\title{
Existence Conditions for a Classical Solution of the Cauchy Problem for the Diffusion-Wave Equation with a Partial Caputo Derivative
}

\author{
A. A. Voroshilov and A. A. Kilbas \\ Presented by Academician S.M. Nikol'skii November 22, 2006
}

Received November 23, 2006

DOI: $10.1134 / \mathrm{S} 1064562407030209$

Consider the Cauchy problem

$$
\begin{gathered}
\left({ }^{c} D_{0+, t}^{\alpha} u\right)(x, t)=\lambda^{2} \Delta_{x} u(x, t), \quad x \in \mathbf{R}^{m}, \\
t>0, \quad \alpha>0, \quad \lambda>0, \\
\frac{\partial^{k} u}{\partial t^{k}}(x, 0+)=f_{k}(x), \quad k=0,1, \ldots, n-1, \\
n=-[-\alpha], \quad x \in \mathbf{R}^{m} .
\end{gathered}
$$

Here, $\left({ }^{c} D_{0+, t}^{\alpha} u\right)(x, t)$ is the $\alpha$ th-order Caputo derivative of $u(x, t)$ with respect to the second argument $(\alpha>0)$ [1, Section 2.4.1],

$$
\begin{gathered}
\left({ }^{c} D_{0+, t}^{\alpha} u\right)(x, t) \\
=\left(\frac{\partial}{\partial x}\right)^{n} \frac{1}{\Gamma(n-\alpha)} \int_{0} \frac{u(x, \tau)-\sum_{k=0}^{n-1} \frac{\tau^{k}}{k !} \frac{\partial^{k} u}{\partial \tau^{k}}(x, 0)}{(t-\tau)^{\alpha-n+1}} d \tau, \\
n=-[-\alpha],
\end{gathered}
$$

and $\Delta_{x}$ is the Laplacian with respect to the first argument $x=\left(x_{1}, x_{2}, \ldots, x_{m}\right) \in \mathbf{R}^{m}: \Delta_{x}=\sum_{j=1}^{m} \frac{\partial^{2} u}{\partial x_{j}^{2}}$. For $\alpha=1$ and $\alpha=2$, Eq. (1) coincides with the heat (diffusion) equation and the wave equation, respectively. That is why Eq. (1) is known as the diffusion-wave equation [1, p. 146].

A function $u(x, t)\left(x \in \mathbf{R}^{m}, t>0\right)$ is a classical solution to Cauchy problem (1), (2) if (i) $u(x, t)$ is twice continuously differentiable with respect to $x$ for every $t>0$;

Faculty of Mechanics and Mathematics, Belarusian State University, pr. Nezavisimosti 4, Minsk, 220050 Belarus e-mail: kilbas@bsu.by (ii) for every $x \in \mathbf{R}^{m}, u(x, t)$ is a continuous function of $t$ and has a continuous partial derivative of order $\alpha$ with respect to $t$; and (iii) Eqs. (1) and (2) hold true.

We examine the existence conditions for a classical solution to problem (1), (2) when $0<\alpha<2$. Specifically, our study is based on the scheme proposed in [2] for analyzing the Cauchy problem with a regularized fractional derivative of order $0<\alpha<1$.

It was shown in [3] that problem (1), (2) with $0<\alpha \leq 1$ and $1<\alpha<2$ has the explicit solutions

$$
\begin{gathered}
u(x, t)=\int_{\mathbf{R}^{m}} G_{1}^{\alpha}(x-\tau, t) f_{0}(\tau) d \tau, \quad 0<\alpha \leq 1, \\
u(x, t) \\
=\int_{\mathbf{R}^{m}}\left[{ }^{c} G_{1}^{\alpha}(x-\tau, t) f_{0}(\tau)+{ }^{c} G_{2}^{\alpha}(x-\tau, t) f_{1}(\tau)\right] d \tau, \\
1<\alpha<2,
\end{gathered}
$$

respectively. Here,

$$
\begin{gathered}
{ }^{c} G_{k}^{\alpha}(x, t)=\frac{2^{-m}|x|^{1-m / 2}}{\lambda^{1+m / 2} \pi^{(m-1) / 2}} t^{k-1-\alpha(m+2) / 4} \\
\times H_{2,2}^{2,0}\left[\frac{|x|}{\lambda} t^{-\alpha / 2} \mid \begin{array}{c}
(m / 4,1 / 2),(k-\alpha(m+2) / 4, \alpha / 2) \\
(m / 2-1,1),(1 / 2-m / 4,1 / 2)
\end{array}\right] \\
k=1,2
\end{gathered}
$$

and $H_{p, q}^{m, n}(z)$ is the so-called H-function (see, e.g., [4, Section 1.1]).

For integers $m, n, p$, and $q$ such that $0 \leq m \leq q$ and $0 \leq$ $n \leq p$; for $a_{i}, b_{j} \in C$; and for $\alpha_{i}, \beta_{j} \in R_{+}=(0, \infty)(i=1$, $2, \ldots, p ; j=1,2, \ldots, q)$, the $\mathrm{H}$-function $H_{p, q}^{m, n}(z)$ is defined by the Mellin-Barns integral 


$$
\begin{aligned}
& H_{p, q}^{m, n}(z) \equiv H_{p, q}^{m, n}\left[z\left[\begin{array}{l}
\left(a_{i}, \alpha_{i}\right)_{1, p} \\
\left(b_{j}, \beta_{j}\right)_{1, q}
\end{array}\right]\right. \\
& =\frac{1}{2 \pi i} \int_{L} \frac{\prod_{j=1}^{m} \Gamma\left(b_{j}+\beta_{j} s\right) \prod_{i=1}^{n} \Gamma\left(1-a_{i}-\alpha_{i} s\right)}{\prod_{i=n+1}^{p} \Gamma\left(a_{i}+\alpha_{i} s\right) \prod_{j=m+1}^{q} \Gamma\left(1-b_{j}-\beta_{j} s\right)} z^{-s} d s .
\end{aligned}
$$

Here,

$$
z^{-s}=\exp [-s\{\ln |z|+i \arg z\}], \quad z \neq 0 ;
$$

the poles

$$
b_{j l}=\frac{-b_{j}-l}{\beta_{j}}, \quad i=1,2, \ldots, m ; \quad l=0,1,2, \ldots
$$

of the gamma functions $\Gamma\left(b_{j}+\beta_{j} s\right)$ do not coincide with the poles

$$
a_{i k}=\frac{1-a_{i}+k}{\alpha_{i}}, i=1,2, \ldots, n ; k=0,1,2, \ldots
$$

of the gamma functions $\Gamma\left(1-a_{i}-\alpha_{i} k\right)$ :

$$
\begin{gathered}
\alpha_{i}\left(b_{j}+l\right) \neq \beta_{j}\left(a_{i}-k-1\right), \quad i=1,2, \ldots, n ; \\
j=1,2, \ldots, m ; \quad k, l=0,1,2, \ldots ;
\end{gathered}
$$

$L$ in (7) is a special infinite contour such that all $b_{j l}$ and $a_{i k}$ lie on the left and right of it, respectively.

By using the properties of $\mathrm{H}$-functions [4, Section 2.1], ${ }^{c} G_{1}^{\alpha}(x, t)$ and ${ }^{c} G_{2}^{\alpha}(x, t)$ can be simplified to

$$
\begin{gathered}
{ }^{c} G_{k}^{\alpha}(x, t) \\
=|x|^{-m} \pi^{-m / 2} t^{k-1} H_{1,2}^{2,0}\left[\frac{|x|^{2}}{4 \lambda^{2} t^{\alpha}} \mid \begin{array}{c}
(k, \alpha) \\
(m / 2,1),(1,1)
\end{array}\right], \\
k=1,2 .
\end{gathered}
$$

To analyze the asymptotics of solutions (4) and (5) at large $x$, we estimate ${ }^{c} G_{k}^{\alpha}(x, t)(k=1,2)$ and their derivatives with respect to $x$.

Below is an asymptotic estimate for the $H_{1,2}^{2,0}$-functions in (10).

Lemma 1. Let $m \in N, 0<\alpha<2, k=1$ for $0<\alpha \leq 1$; and $k=1,2$ for $1<\alpha<2$.

Then, for any fixed $t>0$, we have the asymptotic estimates

$$
H_{1,2}^{2,0}\left[\frac{|x|^{2}}{4 \lambda^{2} t^{\alpha}} \mid \begin{array}{c}
(k, \alpha) \\
(m / 2,1),(1,1)
\end{array}\right]
$$

$$
\begin{gathered}
=A_{k} \exp \left[-(2-\alpha) \alpha^{\alpha /(2-\alpha)}\left(\frac{|x|^{2}}{4 \lambda^{2} t^{\alpha}}\right)^{1 /(2-\alpha)}\right] \\
\times\left(\frac{|x|^{2}}{4 \lambda^{2} t^{\alpha}}\right)^{(m / 2+1-k) /(2-\alpha)}\left[1+O\left(\left(\frac{t^{\alpha}}{|x|^{2}}\right)^{1 /(2-\alpha)}\right)\right], \\
|x| \rightarrow \infty, \quad k=1,2,
\end{gathered}
$$

where $A_{k}$ are constants.

Proof. Formula (11) follows from the exponential behavior at infinity of the more general $\mathrm{H}_{p, q}^{q, 0}$-function as shown in Theorem 1.10 in [4, p. 17].

Formula (11) yields the following asymptotic estimates for ${ }^{c} G_{1}^{\alpha}(x, t)$ and ${ }^{c} G_{2}^{\alpha}(x, t)$ in (4) and (5) for fixed $t>0$ as $|x| \rightarrow \infty$ :

$$
\begin{gathered}
{ }^{c} G_{k}^{\alpha}(x, t) \\
=B_{k} \exp \left[-(2-\alpha) \alpha^{\alpha /(2-\alpha)}\left(\frac{|x|^{2}}{4 \lambda^{2} t^{\alpha}}\right)^{1 /(2-\alpha)}\right]|x|^{-m} t^{k-1} \\
\times\left(\frac{|x|^{2}}{4 \lambda^{2} t^{\alpha}}\right)^{(m / 2+1-k) /(2-\alpha)}\left[1+O\left(\left(\frac{t^{\alpha}}{|x|^{2}}\right)^{1 /(2-\alpha)}\right)\right],(12) \\
|x| \rightarrow \infty,
\end{gathered}
$$

where

$$
B_{k}=\pi^{-m / 2} A_{k}
$$

$k=1$ for $0<\alpha \leq 1$; and $k=1,2$ for $1<\alpha<2$.

Since $0<\alpha<2$, it follows from (12) that, for any fixed $t>0$, the functions ${ }^{c} G_{1}^{\alpha}(x, t)$ and ${ }^{c} G_{2}^{\alpha}(x, t)$ tend to zero as $|x| \rightarrow 0$ :

$$
\lim _{|x| \rightarrow \infty}{ }^{c} G_{1}^{\alpha}(x, t)=\lim _{|x| \rightarrow \infty}{ }^{c} G_{2}^{\alpha}(x, t)=0 .
$$

Let us estimate ${ }^{c} G_{k}^{\alpha}(x, t)(k=1,2)$ for $|x|^{2} t^{-\alpha}<1, x \neq 0$.

According to Theorem 1.12 in [4, p. 20], the asymptotic expansion of the $\mathrm{H}$-function

$$
H_{1,2}^{2,0}\left[z \mid \begin{array}{c}
(k, \alpha) \\
(m / 2,1),(1,1)
\end{array}\right]
$$

at zero is given by the formula

$$
H_{p, q}^{m, n}(z)=\sum_{j}^{\prime}\left[h_{j}^{*} z^{b_{j} / \beta_{j}}+o\left(z^{b_{j} / \beta_{j}}\right)\right]
$$

$+\sum_{j}\left[H_{j}^{*} z^{b_{j} / \beta_{j}}[\ln z]^{N_{j}^{*}-1}+o\left(z^{b_{j} / \beta_{j}}[\ln z]^{N_{j}^{*}-1}\right)\right], \underset{(15)}{z \rightarrow 0}$

where the sums in $\sum^{\prime}$ and $\sum^{\prime \prime}$ are taken over $j(j=1$, $2, \ldots, m)$ such that the gamma functions $\Gamma\left(b_{j}+\beta_{j} s\right)$ in

DOKLADY MATHEMATICS Vol. 75 No. 32007 
(7) have simple poles and poles of order $N_{j}^{*}$, respectively, at the points $b_{j}=b_{j 0}$ defined by (8).

Using formula (15), we derive the following estimates at $z \rightarrow 0$ :

$$
\begin{gathered}
H_{1,2}^{2,0}\left[z \mid \begin{array}{c}
(k, \alpha) \\
(m / 2,1),(1,1)
\end{array}\right] \\
=h_{1}^{*} z^{m / 2}+o\left(z^{m / 2}\right)+h_{2}^{*} z+o(z)
\end{gathered}
$$

for odd $m$;

$$
H_{1,2}^{2,0}\left[z \mid \begin{array}{c}
(k, \alpha) \\
(m / 2,1),(1,1)
\end{array}\right]=H_{1}^{*} z \ln z+o(z \ln z)
$$

for $m=2$; and

$$
\begin{gathered}
H_{1,2}^{2,0}\left[z \mid \begin{array}{c}
(k, \alpha) \\
(m / 2,1),(1,1)
\end{array}\right] \\
=H_{1}^{*} z^{m / 2} \ln z+o\left(z^{m / 2} \ln z\right)+h_{2}^{*} z+o(z)
\end{gathered}
$$

for even $m>2$, where $h_{1}^{*}, h_{2}^{*}$, and $H_{1}^{*}$ are constants.

Formulas (16)-(18) yield estimates of ${ }^{c} G_{k}^{\alpha}(x, t)$ for $|x|^{2} t^{-\alpha}<1, x \neq 0$ :

$$
\begin{gathered}
\left|{ }^{c} G_{k}^{\alpha}(x, t)\right| \leq C t^{k-1-\alpha}|x|^{-m+2}, \quad k=1,2, \\
x \in \mathbf{R}^{m}, \quad m>2, \\
\left|{ }^{c} G_{k}^{\alpha}(x, t)\right| \leq C t^{k-1-\alpha}\left(\left|\ln \left(|x|^{2} t^{-\alpha}\right)\right|+1\right), \\
k=1,2, \quad x \in \mathbf{R}^{2}, \\
\left|{ }^{c} G_{k}^{\alpha}(x, t)\right| \leq C t^{k-1-\alpha / 2}, \quad k=1,2, \quad x \in \mathbf{R} .
\end{gathered}
$$

Inequalities (19)-(21) imply that $\left|G_{1}^{\alpha}(x, t)\right|$ and $\left|G_{2}^{\alpha}(x, t)\right|$ are integrable with respect to $x \in \mathbf{R}^{m}$ at zero for any $t>0$.

Taking into account asymptotics (12) of these functions at infinity, we infer the following assertion.

Lemma 2. Let $m \in N, 0<\alpha<2 ; k=1$ for $0<\alpha \leq 1$; and $k=1,2$ for $1<\alpha<2$.

Then ${ }^{c} G_{k}^{\alpha}(x, t) \in L_{1}\left(\mathbf{R}^{m}\right)$ for any $t_{0}>0$.

The result below follows from Lemmas 1 and 2 .

Theorem 1. Let $0<\alpha \leq 2, m \in N$, and $\lambda>0$.

(a) If $0<\alpha \leq 1$ and $f_{0}(x)$ is bounded and absolutely integrable on $\mathbf{R}^{m}$, then solution (4) to Cauchy problem (1), (2) exists and tends to zero as $|x| \rightarrow \infty$ for any fixed $t>0$.

(b) If $1<\alpha<2$ and if $f_{0}(x)$ and $f_{1}(x)$ are bounded and absolutely integrable on $\mathbf{R}^{m}$, then solution (5) to Cauchy problem (1), (2) exists and tends to zero as $|x| \rightarrow \infty$ for any fixed $t>0$.
Proof. Since ${ }^{c} G_{1}^{\alpha}(x, t)$ is absolutely integrable with respect to $x \in \mathbf{R}^{m}$ for every $t>0$ and $f_{0}(x)$ is bounded on $\mathbf{R}^{m}$, the assertion in [5, p. 532] implies that convolution (4) exists. The rest of the proof is based on direct estimates.

An asymptotic expansion of ${ }^{c} G_{k}^{\alpha}(x, t)$ as $|x|^{2} t^{-\alpha} \rightarrow \infty$ and $|x|^{2} t^{-\alpha} \rightarrow 0$ is given by formulas (12) and (19)-(21), respectively. The derivatives of these functions can be found as follows. Introducing $\rho=|x|$ and using formula (2.2.2) from [4] for the derivatives of $\mathrm{H}$-functions, we obtain

$$
\begin{gathered}
D_{\rho}^{n}\left({ }^{c} G_{k}^{\alpha}(x, t)\right) \\
=D_{\rho}^{n}\left(\rho^{-m} \pi^{-m / 2} t^{k-1} H_{1,2}^{2,0}\left[\frac{\rho^{2}}{4 \lambda^{2} t^{\alpha}} \mid \begin{array}{c}
(k, \alpha) \\
(m / 2,1),(1,1)
\end{array}\right]\right) \\
=(-1)^{n} \rho^{-m-n} \pi^{-m / 2} t^{k-1} \\
\times H_{2,3}^{3,0}\left[\frac{\rho^{2}}{4 \lambda^{2} t^{\alpha}} \mid \begin{array}{c}
(k, \alpha),(m, 2) \\
(m+n, 2),(m / 2,1),(1,1)
\end{array}\right] ; \quad \\
\rho>0, t>0, n=1,2,3 .
\end{gathered}
$$

According to Theorem 1.10 in $[4, p .17]$ on the exponential behavior of an $\mathrm{H}$-function at infinity, it follows from (22) that

$$
\begin{gathered}
D_{x}^{n}\left({ }^{c} G_{k}^{\alpha}(x, t)\right) \\
=B_{k} \exp \left[-(2-\alpha) \alpha^{\alpha /(2-\alpha)}\left(\frac{\rho^{2}}{4 \lambda^{2} t^{\alpha}}\right)^{1 /(2-\alpha)}\right] \\
\times \rho^{-m} t^{k-1}\left(\frac{\rho^{2}}{4 \lambda^{2} t^{\alpha}}\right)^{(m / 2+n-k+1) /(2-\alpha)} \\
\times\left[1+O\left(\left(\frac{t^{\alpha}}{\rho^{2}}\right)^{1 /(2-\alpha)}\right)\right], \quad \rho^{2} t^{-\alpha} \rightarrow \infty .
\end{gathered}
$$

The asymptotics of $D_{\rho}^{n}\left({ }^{c} G_{k}^{\alpha}(\rho, t)\right)$ as $\rho \rightarrow 0$ can be found as follows. According to (15) and (22), for $\rho^{2} t^{-\alpha}<1, \rho>0, t>0$, and $n \geq 1$, we have

$$
\begin{gathered}
\left|D_{x}^{n} G_{k}^{\alpha}(x, t)\right| \leq C t^{-k}|x|^{-m-n+2}, \\
k=1,2, \quad m \geq 2, \quad C>0 .
\end{gathered}
$$

To derive an estimate at $m=1$, we use the properties of H-functions to transform ${ }^{c} G_{k}^{\alpha}(x, t)$ into

$$
{ }^{c} G_{k}^{\alpha}(x, t)=\frac{1}{2 \lambda} t^{k-1-\alpha / 2} H_{1,1}^{1,0}\left[\frac{|x|}{\lambda t^{\alpha / 2}} \mid \begin{array}{c}
(k-\alpha / 2, \alpha / 2) \\
(0,1)
\end{array}\right] .
$$


According to formula (2.2.2) in [4],

$$
\begin{gathered}
D_{x}^{n}\left({ }^{c} G_{k}^{\alpha}(x, t)\right)=\frac{(-1)^{n}}{2 \lambda} t^{k-1-\alpha / 2}|x|^{-n} \\
\times H_{2,2}^{2,0}\left[\frac{|x|}{\lambda t^{\alpha / 2}} \mid \begin{array}{c}
(k-\alpha / 2, \alpha / 2),(0,1) \\
(n, 1),(0,1)
\end{array}\right] \\
=\frac{(-1)^{n}}{2 \lambda} t^{k-1-\alpha / 2}|x|^{-n} H_{1,1}^{1,0}\left[\frac{|x|}{\lambda t^{\alpha / 2}} \mid \begin{array}{c}
(k-\alpha / 2, \alpha / 2) \\
(n, 1)
\end{array}\right] .
\end{gathered}
$$

Using (15) for $|x|^{2} t^{-\alpha}<1, x \neq 0, n \geq 1$, we obtain

$$
\left|D_{x}^{n}\left({ }^{c} G_{k}^{\alpha}(x, t)\right)\right| \leq C t^{k-1-(n+1) \alpha / 2} .
$$

Combining estimates (12), (19)-(21), and (23)-(25) yields the following result.

Theorem 2. (a) Suppose that $f_{0}(x)$ is continuous on $\mathbf{R}^{m}$; increases exponentially at infinity,

$$
\left|f_{0}(x)\right| \leq C \exp \left(h|x|^{\mu}\right), C>0, h>0, \mu<\frac{2}{2-\alpha} ;
$$

and, additionally, is locally Hölder for $m>1$. Let ${ }^{c} G_{1}^{\alpha}(x, t)$ be defined by formula (10) with $k=1$.

Then function (4) is a classical solution to problem (1), (2) with $0<\alpha \leq 1$; moreover, $u(x, t) \in C[0, \infty)$ for any $x \in \mathbf{R}^{m}$.

(b) Let $f_{0}(x)$ and $f_{1}(x)$ be continuous on $\mathbf{R}^{m}$, satisfy estimate (26), and be locally Hölder for $m>1$; and let
${ }^{c} G_{1}^{\alpha}(x, t)$ and ${ }^{c} G_{2}^{\alpha}(x, t)$ be defined by formula (10) with $k=1$ and $k=2$, respectively.

Then function (5) is a classical solution to problem (1), (2) with $1<\alpha<2$ and $u(x, t) \in C[0, \infty)$ for any $x \in \mathbf{R}^{m}$.

\section{ACKNOWLEDGMENTS}

The authors are grateful to the participants of the seminar held at the Department of Function Theory of the Steklov Institute of Mathematics, Russian Academy of Sciences (supervised by Academician S.M. Nikol'skii and Corresponding Members O.V. Besov and L.D. Kudryavtsev) for a helpful discussion of these results. This work was supported by the Foundation for Basic Research of the Belarus Republic, project nos. F05MS-050 and F06R-106.

\section{REFERENCES}

1. I. Podlubny, Fractional Differential Equations (Academic, San Diego, 1999).

2. A. N. Kochubei, Differ. Uravn. 26, 660-670 (1990).

3. A. A. Voroshilov and A. A. Kilbas, Differ. Equations 42, 638-649 (2006) [Differ. Uravn. 42, 599-609 (2006)].

4. A. A. Kilbas and M. Saigo, H-Transforms (Chapman \& Hall/CRC, Boca Raton, FL, 2004).

5. V. A. Zorich, Mathematical Analysis (MTsNMO, Moscow, 2002), Vol. 2 [in Russian]. 